\title{
Regulación de Voltaje por Control de
Disparo Empleando Tecnologías FPAA
}

\author{
Voltage Regulation by Phase \\ Commutation Control Running On \\ FPAA
}

.

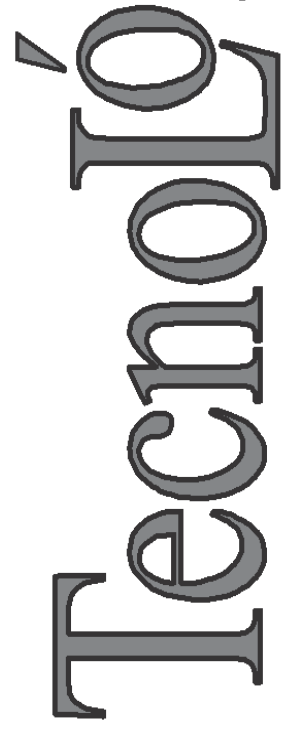

Ckristian R. Durán-Blanco ${ }^{1}$

Julio A. Flórez-Vargas ${ }^{2}$

Ricardo Alzate-Castaño ${ }^{3}$

1 Escuela de Ingenierías Eléctrica, Electrónica y de

Telecomunicaciones (E3T),

Universidad Industrial de Santander,

Bucaramanga-Colombia

2 Escuela de Ingenierías Eléctrica, Electrónica y de

Telecomunicaciones (E3T),

Universidad Industrial de Santander,

Bucaramanga-Colombia

3 Escuela de Ingenierías Eléctrica, Electrónica y de

Telecomunicaciones (E3T),

Universidad Industrial de Santander,

Bucaramanga-Colombia

ralzatec@uis.edu.co 


\section{Resumen}

El presente artículo presenta resultados de implementación de algoritmos de control de disparo sobre un circuito convertidor de potencia del tipo rectificador controlado. La novedad de la implementación está constituida por la plataforma de ejecución basada en tecnología de arreglos analógicos programables por campo FPAA. Se verifica la acción de técnicas de control proporcional, PID y de modos deslizantes para regular la tensión de salida del circuito hacia una carga (bombilla) ante la acción de perturbaciones. Los resultados obtenidos permiten mostrar la potencialidad de la tecnología de implementación propuesta para aplicaciones de circuitos reconfigurables en electrónica de potencia.

\section{Palabras clave}

Circuitos reconfigurables; control deslizante; electrónica de potencia; FPAA; rectificador controlado.

\section{Abstract}

In this article we present a control scheme for triggering a SCR-based rectifier circuit. As implementation platform we use a fieldprogrammable-analog-array device (FPAA) that allows the development of algorithms for proportional, PID and sliding mode (SMC) control techniques. Results show good performance of tested techniques to avoid effects of perturbations over the circuit load, by keeping regulated the output voltage. This suggests the potential use of the proposed technology for applications of reconfigurable hardware in power electronics.

\section{Keywords}

FPAA; power electronics; reconfigurable hardware; SCR-based rectifier circuit; sliding mode control. 


\section{INTRODUCCIÓN}

En el mundo actual la dependencia de la electricidad aumenta a niveles insostenibles. Como consecuencia directa de ello se estimula a nivel gubernamental la exploración de fuentes alternativas de energía y el uso racional de la misma (URE) a partir de la apropiación y el desarrollo de herramientas tecnológicas que, por tanto, constituyen un tópico de marcada pertinencia para los diferentes campos de aplicación de la ingeniería. En este contexto, se destaca el interés por mejorar las características de eficiencia en los sistemas de generación de energía eléctrica sobre todo para casos en los cuales la energía proviene de fuentes alternativas como el viento (Burton \& Davies, 1996) o la luz solar (Alvarez \& Arce, 2003).

Estos sistemas de generación alternativa pueden participar activamente como insumo en el sistema interconectado inteligente o Smart-grid (Shaaban, 2012). La estructura típica de un sistema de microgeneración eléctrica basado en energías alternativas posee como actor principal al circuito convertidor de potencia, el cual recibe una señal generalmente tenue o de niveles inapropiados, y entrega hacia la carga los niveles de corriente y voltaje requeridos a partir del denominado proceso de conversión (Rashid, 1993). Las formas de onda a la salida del sistema de microgeneración son entonces el elemento crucial para valorar el desempeño del convertidor de potencia. Por tanto, el comportamiento del circuito puede describirse a partir de su evolución temporal transitoria y estacionaria dando cabida al análisis y el diseño de técnicas de control para manipular la dinámica del sistema de conversión de potencia a partir del control de activación (o disparo) de los dispositivos de conmutación asociados a la topología particular considerada (Alves de Melo \& Bento, 2009; Jezernik, 2010; Yang \& Wang, 2011; Iannelli, 2005; Sepulchre \& Kokotovic, 1996; Scuadroni, 2002; RuizCruz et al., 2012).

Una herramienta fundamental para llevar a la práctica estrategias de control está representada por plataformas prestantes de hardware programable que incluyen principalmente microcontroladores de alta gama, procesadores digitales de señal (DSPs) y arreglos digitales programables (FPGAs). Sin embargo, existen 
nuevas alternativas como los arreglos analógicos programables (FPAAs - field programmable analog arrays) que poseen capacidades similares de reconfiguración que sus contrapartes digitales (Anadigm, 2003; Gyorok, 2009; Schlottmann \& Hasler, 2011; Visan et al., 2011). Tomando en cuenta las anteriores consideraciones, el presente artículo propone la implementación de técnicas de control convencional y no-convencional sobre un FPAA para controlar el disparo de un dispositivo de conmutación como parte de un rectificador controlado, buscando aplicación en el contexto de los circuitos convertidores de potencia.

El contenido del artículo se presenta como sigue: la sección 1 motivó las ideas del contexto temático; en la sección 2 se realiza una descripción del sistema implementado; en la sección 3 se ilustra la metodología desarrollada para verificar las técnicas de control propuestas; en la sección 4 se realiza una discusión de los resultados obtenidos a partir de los cual se generan conclusiones generales en la sección 5.

\section{EL SISTEMA}

La Fig. 1 ilustra el diagrama de bloques general del sistema implementado para verificar las acciones de control ejecutadas sobre un FPAA para regular los niveles de tensión a la salida de un rectificador controlado.

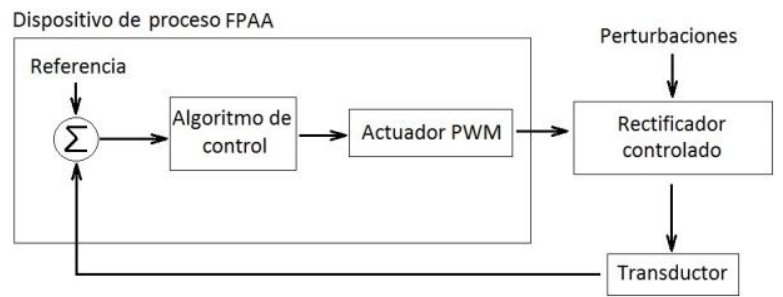

Fig. 1. Diagrama de bloques para el sistema implementado. Fuente: Autores

A continuación se describen los aspectos relevantes que caracterizan la estructura y el funcionamiento de cada uno de los bloques respectivos. 


\subsection{Dispositivo de Proceso}

Para ejecutar el procesamiento de las señales se empleó un sistema de desarrollo ANADIGM ${ }^{\circledR}$ AN221K04 basado en el FPAA AN120E04. Este dispositivo posee entre otras características: interfaces de comunicación USB y SPI, un oscilador de $16 \mathrm{MHz}$ y puertos configurables de entrada y salida. La programación del sistema se realiza en modo gráfico empleando la herramienta ANADIGM-Designer ${ }^{\circledR}$. Las rutinas fueron constituidas a partir de tres bloques principales: un punto de suma, un generador de PWM y el respectivo subsistema controlador. Un ejemplo de la interfaz de programación empleada se presenta en la Fig. 2.

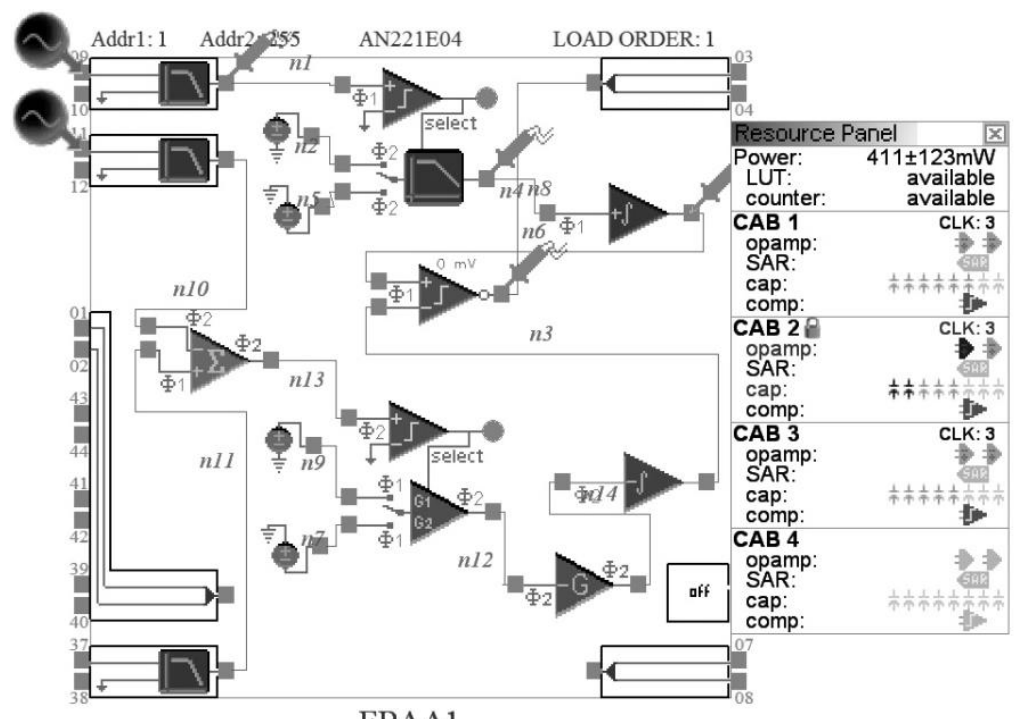

FPAA1

Fig. 2. Ilustración interfaz de programación gráfica para dispositivo de proceso. Fuente: Autores

\subsection{Rectificador Controlado}

El diagrama esquemático del circuito sobre el cual se implementó el control de disparo se muestra en la Fig. 3. Dicho circuito corresponde con un rectificador controlado de media onda que 
emplea como dispositivo de conmutación un SCR de referencia C106D. El voltaje aplicado en su entrada posee aproximadamente 6.5 Vpico / 60 [Hz]. Como salida por tanto se busca obtener un semi-ciclo de la onda sinusoidal de entrada el cual, para el caso de una conducción completa $\left(180^{\circ}\right)$ permitirá alrededor de 4.6 VDC de voltaje eficaz aplicado a la carga, una bombilla de 12 VDC con impedancia nominal medida de $100[\Omega]$. El objetivo es por tanto seleccionar un ángulo de disparo entre $0^{\circ}$ y $180^{\circ}$, para obtener los niveles de energía requeridos en la salida. La Fig. 4 muestra las formas de onda generadas como salida del rectificador controlado para ángulos de disparo dados respectivamente por $0^{\circ}, 90^{\circ}$ y $175^{\circ}$.

\subsection{Transductor}

Tomando en cuenta que la variable a controlar en el esquema de control corresponde al voltaje DC en la carga (bombilla), se realizó una medida de la diferencia de potencial en los terminales del dispositivo. Considerando el carácter pulsante de la señal entregada por el rectificador controlado (ver Fig. 4), fue necesario implementar un filtro pasa-bajas para obtener la componente fundamental (DC) de la señal de interés o medida. La Fig. 5 ilustra un ejemplo del tipo de señal obtenida a la salida del filtro, la cual es posteriormente acoplada como entrada analógica en el dispositivo de proceso.

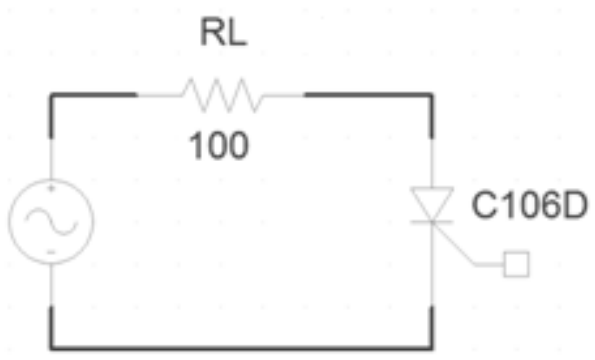

Fig. 3. Diagrama esquemático para rectificador controlado por disparo de SCR.

Fuente: Autores 


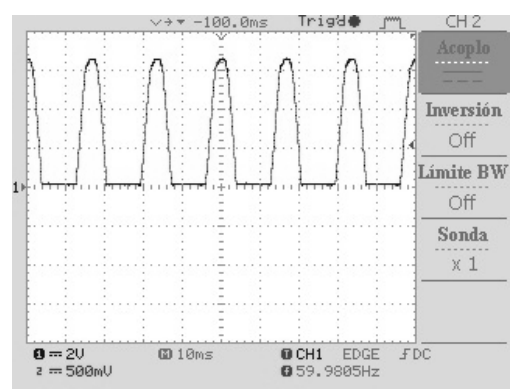

a)

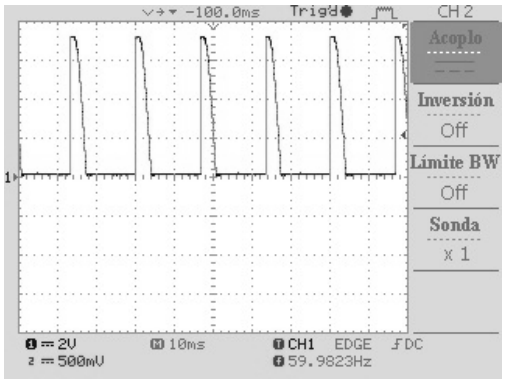

b)

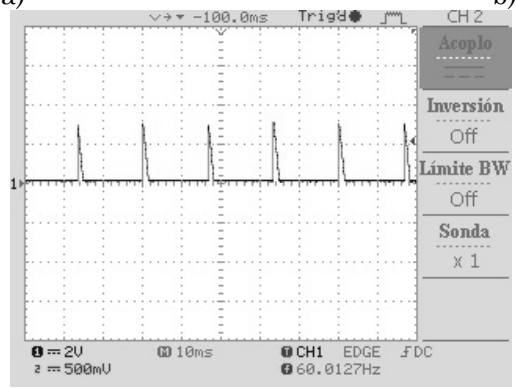

c)

Fig. 4. Señal de salida del rectificador para disparo en: a) $0^{\circ}$, b) $90^{\circ}$ y c) $175^{\circ}$. Fuente: Autores

\subsection{Perturbaciones}

Para verificar el efecto de la acción de control sobre el sistema, se incorporó una carga en serie a la bombilla, con las mismas características de la nominal. El método de activación para la perturbación se realizó de manera manual mediante un interruptor. La Fig. 5 permite verificar la variación sobre el nivel de tensión DC medido en la carga tras aplicar la perturbación mencionada sobre el circuito.

\subsection{Sistema Experimental Definitivo}

La Fig. 6 permite visualizar el sistema experimental constituido para verificar la acción de controladores implementados sobre FPAA para regular los niveles de tensión entregados a una carga 
(bombilla) por parte de un circuito convertidor de potencia correspondiente con un rectificador controlado.

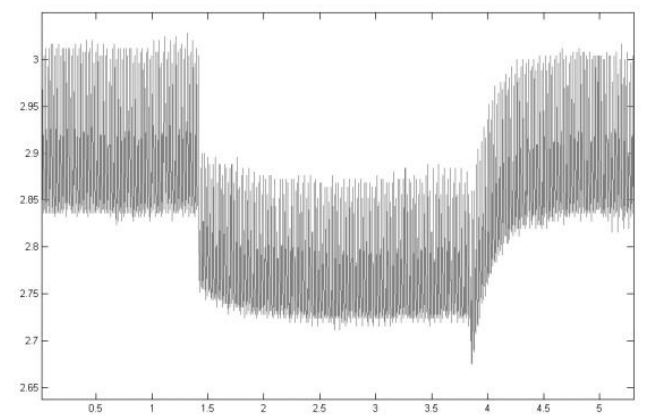

Fig. 5. Medida de tensión en la carga afectada por acción de perturbaciones. Tensión de salida vs. tiempo. Fuente: Autores

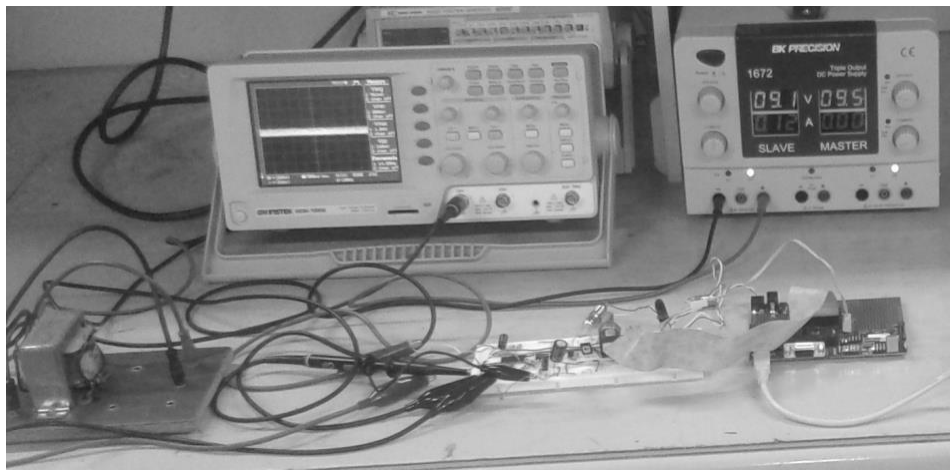

Fig. 6. Prototipo experimental final. Fuente: Autores

\section{METODOLOGÍA EXPERIMENTAL}

Una vez constituido el sistema experimental, se realizaron sobre el mismo un conjunto de pruebas consistentes en la visualización del comportamiento en el tiempo para las señales de respuesta (nivel DC a la salida del filtro) ante la incidencia de perturbaciones, operando sobre diferentes acciones de control sobre el error. El error de medida se determinó mediante el ángulo de 
disparo; es decir, tomando en cuenta que el voltaje promedio a la salida del circuito $V_{o}(t)$, se define a partir de (1),

$V_{o}(t)=\frac{1}{2 \pi} \int_{\alpha(t)}^{\pi} V_{s} \sin (\theta) d \theta=\frac{V_{s}}{2 \pi}(\cos (\alpha(t))+1) \alpha<\pi$

siendo $a(t)$ el ángulo de disparo del SCR y $V_{s}$ el voltaje de entrada, para un $V_{o}(t)$ deseado existe un $\alpha(t)$ de referencia generado por el circuito de disparo en el dispositivo de proceso. Si existen alteraciones (perturbaciones) en el sistema, el valor medido y el valor deseado presentarán diferencias (error de medida) que deberán corregirse mediante una acción de control. La Fig. 5 ilustró el efecto sobre el circuito al aplicar perturbaciones en la carga. Como estrategia comparativa, se realizaron verificaciones de acciones de control de tipo proporcional, PID y por modos deslizantes. En esta última, la señal de control $u(t)$ se determinó mediante (2).

$u(t)=k * \operatorname{sgn}($ error $)$

siendo $k$ una ganancia de tipo constante y $\operatorname{sgn}($.$) la función$ signo, la cual posee valores binarios (+1 ó -1) dependiendo del signo de su argumento, en este caso el error de medida.

\section{RESULTADOS Y DISCUSIÓN}

En la Fig. 7 se presentan los resultados de implementación para el caso proporcional. De los mismos se observa el efecto de atenuación sobre las perturbaciones al cerrar el lazo de control tras comparar con la Fig. 5. También se evidencia el mejoramiento del desempeño transitorio en la acción de control al incrementarse la ganancia proporcional. Sin embargo aún persiste un error en el valor de estado estable. La Fig. 8 incluye los resultados de la acción PID. De ella se observa una alta incidencia de oscilaciones debidas a las acciones derivativa e integral, que corrigen el error en estado estacionario y que permiten -para el caso b)- atenuar de manera significativa el efecto de la perturbación. Finalmente, la Fig. 9 muestra los resultados para el control por modos deslizan- 

FPAA

tes, a partir de lo cual se nota un mejor desempeño general que se incrementa con el valor asignado a la ganancia $k$. Esta técnica por definición puede asociarse con un control del tipo encendidoapagado y por tanto corresponde con un caso especial del control proporcional en el cual las ganancias se consideran altas y producen saturación de la señal de control.

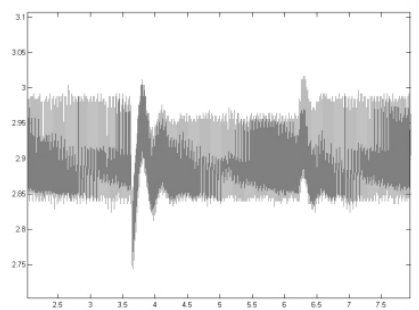

a)

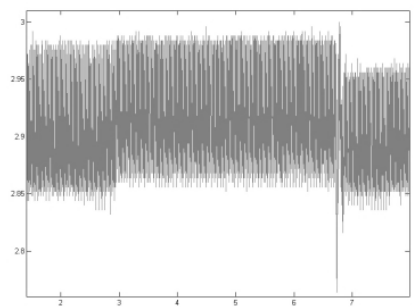

b)

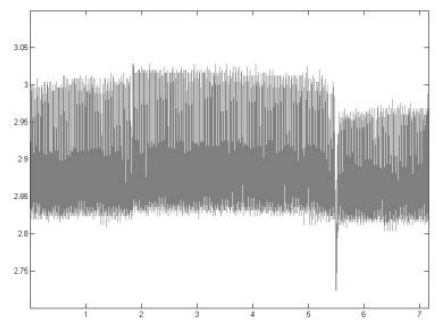

c)

Fig. 7. Respuesta control proporcional: $\mathrm{Kp}=\mathrm{a})$ 0.1, b) 1 y c) 32 . Tensión de salida vs. tiempo. Fuente: Autores

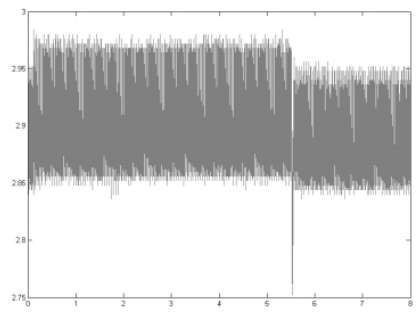

a)

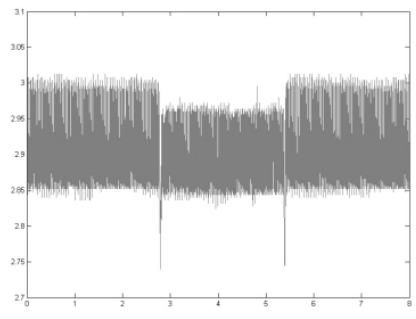

b) 


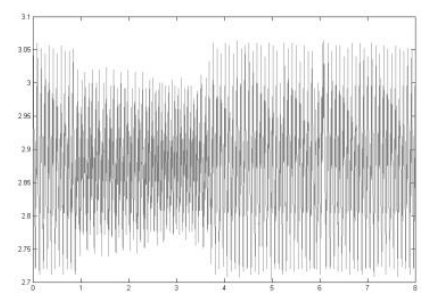

c)

Fig. 8. Respuesta control PID: $(\mathrm{Kp}, \mathrm{Ki}, \mathrm{Kd})=\mathrm{a})(1,0.04,0.04)$, b) $(1,0.25,0.25)$ у c) $(6.4,0.004,0.004)$. Tensión de salida vs. tiempo. Fuente: Autores

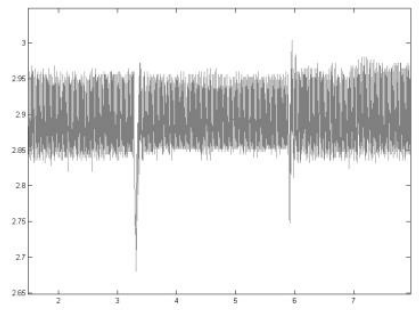

a)

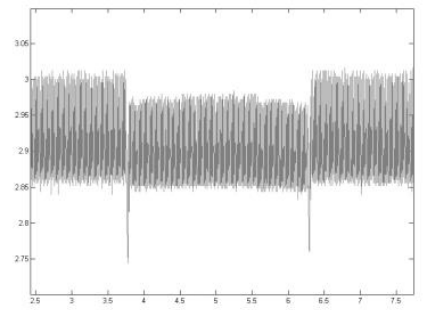

b)

Fig. 9. Respuesta control deslizante: $\mathrm{k}=\mathrm{a}) 0.1 \mathrm{y}$ b) 1 .

Tensión de salida vs. tiempo. Fuente: Autores

\section{CONCLUSIONES}

A partir de los resultados obtenidos, se proponen las siguientes conclusiones: Se implementó un esquema de control de disparo sobre un circuito convertidor de potencia del tipo rectificador controlado. Como plataforma de implementación se utilizó una tarjeta de arreglos analógicos programables por campo FPAA.

Se pudo observar comparativamente el desempeño de controladores de tipo proporcional, PID y por modos deslizantes, para regular la tensión entregada a una carga (bombilla) ante la acción de perturbaciones. Lo anterior permitió verificar el potencial de uso para circuitos analógicos configurables basados en tecnologías FPAA en aplicaciones de electrónica de potencia. Actualmente se verifica la posibilidad de aplicar los desarrollos presentados en un 
prototipo de laboratorio para sistema de microgeneración eléctrica fotovoltaico de 120 [w].

\section{AGRADECIMIENTOS}

Los autores desean agradecer a la Universidad Industrial de Santander por financiar este trabajo a través del proyecto de investigación identificado con el código VIE-UIS 5568.

\section{REFERENCIAS}

Alvarez, G.; Arce, J.; Lira, L.; Heras, M.R. Thermal Performance of an Air Solar Collector with an Absorber Plate Made of Disposable Aluminum Cans. Submitted and accepted to Solar Energy. Journal, in press, 2003.

Alves de Melo A., Bento, S. M., IEEE, and Edison Roberto Cabral da Silva, Fellow, IEEE (2009). "Hybrid One-Cycle Controller for Boost PFC Rectifier." IEEE TRANSACTIONS ON INDUSTRY APPLICATIONS. Vol. 45, No. 1.

Anadigm Inc (2003). AN221E04 datasheet dynamically reconfigurable FPAA with enhanced I/O. Technical report.

Burton J.D., Davies D.G. (1996). Dynamic model of a wind-driven lift pump, Proc. Instn. Mech. Engrs., Vol 210.

Gyorok G. (2009). The FPAA realization of analog robust electronic circuits. IEEE 7th International Conference on Computational Cybernetics. November 26-29. Palma de Mallorca, Spain.

Iannelli, L. (2005). Dithering for sliding mode control of DC/DC converter. Volumen 2 (Power Electronics Specialists Conference, 2004. PESC 04. 2004 IEEE 35th Annual): 1616 - 1620.

Jezernik, K. H., Robert. (2010). "FPGA Hybrid Controller for Unity Power Factor." 14th International Power Electronics and Motion Control Conference, EPE-PEMC 2010.

Rashid M. H., Electrónica de potencia. Circuitos, dispositivos y aplicaciones, México, Prentice Hall Hispanoamericana S.A., 1993.

Rodolphe Sepulchre, M. J., Petar Kokotovic (1996). Constructive nonlinear control. University of California, Santa Barbara, Center for Control Engineering and Computation.

Ruiz-Cruz, R.; Sanchez, E.N.; Ornelas-Tellez, F.; Loukianov, A.G.; Harley, R.G., (2012). Particle Swarm Optimization for Discrete-Time Inverse 
Optimal Control of a Doubly Fed Induction Generator. Cybernetics, IEEE Transactions on , vol.PP, no.99, pp.1,12.

Schlottmann Craig R., \& Hasler Paul E. (2011). A highly dense, low power, programmable analog vector-matrix multiplier: the FPAA implementation. IEEE Journal on emerging and selected topics in circuits and systems, Vol 1, $N^{\circ} 3$, September.

Scuadroni, G. T. (2002). Introducción a los sistemas disipativos y prueba del Teorema de pequeña ganancia. Montevideo - Uruguay., Universidad de la República. Page(s): 14.

Shaaban., J. O. P. M. (2012). Overcoming Challenges of Renewable Energy on Future Smart Grid. TELKOMNIKA, Vol.10, No.2, June 2012, pp. 229 234.

Visan, D.A.; Lita, I.; Cioc, I.B. (2011), Temperature control system based on adaptive PID algorithm implemented in FPAA. Electronics Technology (ISSE), 2011 34th International Spring Seminar on , vol., no., pp.501,504, 11-15 May.

Yang G., Wang J. (2011). "Modeling, Analysis, and Control for the Rectifier of Hybrid HVdc Systems for DFIG-Based Wind Farms" Energy Conversion, IEEE Transactions on. Volumen 26 (1). 